\title{
Galaxy clusters at low redshift
}

\author{
Michael L. Balogh \\ Department of Physics, University of Durham, South Road, Durham UK, DH1 3LE email: \\ M.L.Balogh@Durham.ac.uk
}

\begin{abstract}
We present a new analysis of galaxy colours and star formation rates as a function of environment, from the Sloan Digital Sky Survey and the 2dF Galaxy Redshift Survey. The galaxy distribution is well characterised by only two distinct populations: one that is blue and star-forming, and one that is red and quiescent. The colour and $\mathrm{H} \alpha$ distribution of each of these populations is approximately independent of environment, while their relative abundances depend strongly on local galaxy density. Even in the lowest-density environments, a substantial ( 30 per cent) population of galaxies have red colours and exhibt no detectable $\mathrm{H} \alpha$ emission. We compare these data with theoretical predictions of galaxy formation, properly accounting for observational selection biasses and uncertainties. We conclude that galaxy transformations must either be rapid (with a timescale $\tau \lesssim 0.5 \mathrm{Gyr}$ ), or must occur preferentially at high redshift.
\end{abstract}

\section{Introduction}

It has been known for a long time that many (perhaps most) galaxy properties in the nearby Universe correlate strongly with their environment. Early work by Dressler (1980) and Postman \& Geller (1984) showed that the distribution of galaxy morphologies depends strongly on local galaxy density, in the sense that early-type galaxies (E/S0) dominate in locally dense environments, while late-types (spiral and irregular galaxies) become increasingly important toward lower densities. An important result of this work was that the correlation is strongest with estimates of the local density, with little or no further dependence on the cluster type (i.e. degree of central concentration, or velocity dispersion of the system). In particular, Postman \& Geller (1984) showed that the trend exists in small galaxy groups, as well as in clusters. Although there may be additional effects at work in the very cores of clusters (e.g. Whitmore et al. 1993; Domínguez et al. 2002), it is clear that most of the correlation is driven by the local density of galaxies (or, perhaps, dark matter: Gray et al. 2004, and these proceedings).

It remains an open question to what extent the different galaxy populations in clusters are due to the direct influence of their environment, and how much of it is due to factors related to their mass, or formation time. Part of the reason for this uncertainty is that even in the lowest density environments studied by Dressler (1980), the distribution of galaxy morphologies still differs markedly from the global average; studies of cluster outskirts are therefore required to connect the two environments, where one might expect galaxy transformations may be taking place (Treu et al. 2003, and these proceedings). The advent of large redshift surveys like the Sloan Digital Sky Survey (SDSS, York et al. 2000) and 2dF Galaxy Redshift Survey (2dFGRS, Colless et al. 2003) make it possible to do this at low redshift for the first time.

Unfortunately, galaxy morphology is notoriously difficult to quantify in an objective manner; in particular, no automated method has proven effective at distinguishing elliptical galaxies from S0 galaxies. Nor is it clear that morphology is in any way the fundamental parameter of interest, as it correlates strongly with luminosity (Marzke et al. 1994; Cuesta-Bolao \& Serna 2003), colour and SFR (Kennicutt 1992), among other 
quantities. Since the latter properties are both easier to measure and easier to relate to theoretical predictions, more studies have focussed on the environmental dependence of these properties, most notably galaxy colours (e.g. Butcher \& Oemler 1984; Kodama \& Bower 2001) star formation rates (SFRs, e.g. Balogh et al. 1997) and HI content (Solanes et al. 2001b; van Gorkom 2004, see also contributions from M. Verheijen and J. Solanes, in this volume). These studies and many more have shown that galaxies in dense environments tend to be redder, with lower current star formation rates and low amounts of HI gas for their mass. Although these trends are partly consistent with the morphologydensity relation, there is evidence that galaxies of a fixed morphological type have lower star formation rates (Balogh et al. 1998; Poggianti et al. 1999) and less neutral hydrogen (Solanes et al. 2001a) in clusters. In general these trends vary continuously with local density, with little or no dependence on larger scale structures. For example, there is little or no trend in the global fraction of blue galaxies with cluster velocity dispersion (De Propris et al. 2004; Margoniner et al. 2001).

These trends suggest that galaxies in dense environments are starved of the raw materials needed to make new stars (i.e. cold gas). Many mechanisms have been proposed to cause this, including ram-pressure stripping (Gunn \& Gott 1972), galaxy harassment (Moore et al. 1999) and strangulation (the stripping of a hot gas halo, Larson et al. 1980; Balogh et al. 2000). In particular, the strong evolution seen in the cluster population suggests such processes may have occurred in the recent past (Dressler et al. 1997, and these proceedings). Further clues lie in the exceptions to the general trend of low activity in dense environments. For example, Moss \& Whittle $(1993,2000)$ find that early-type spiral galaxies in clusters actually show stronger $\mathrm{H} \alpha$ emission than galaxies of the same morphological type in the field. Far-infrared observations suggest that some clusters may contain a population of dusty starburst galaxies in excess of that expected in the field (Duc et al. 2002, and contribution to these proceedings), and the presence of $\mathrm{k}+\mathrm{a}$ galaxies in the Coma cluster are also indicative of recent starbursts that may have been induced through interactions with neighbouring galaxies or the cluster gas (Poggianti et al. 2004, and contribution to these proceedings). These observations suggest that interactions may temporarily enhance star formation, leading to rapid consumption of cold gas. Ultimately (over $\sim 1$ Gyr timescales), the net result is a lack of fuel and a low star formation rate.

Until recently, most comprehensive cluster studies have been at moderate redshift, where the smaller angular size of the virialised cluster region facilitates full coverage. The 2dFGRS and SDSS now make it possible to study clusters over arbitrarily wide fields at low redshift, and we have taken advantage of this to trace the trend in $\mathrm{H} \alpha$ emission line strength (directly related to SFR) over the full dynamic range of galaxy densities in the local Universe (Lewis et al. 2002; Gomez et al. 2003). Here, the local density $\Sigma_{5}$ is based on the projected distance to the fifth-nearest neighbour, within $\pm 1000 \mathrm{~km} \mathrm{~s}^{-1}$ and brighter than $M_{r} \sim-20$. The average emission line strength was observed to increase strongly with decreasing $\Sigma_{5}$, in a way holds both within clusters as well as outside them. The trend shows evidence for a critical density at $\Sigma_{5} \sim 1 \mathrm{Mpc}^{-2}$, below which the correlation with environment is much weaker. This density is typical of cluster infall regions, and the observed break suggests that there may be additional physics at work in these environments. Here, we report on a continuation of this work, based on an analysis of colour and SFR distributions in the 2dFGRS and SDSS, and make some initial comparisons with simple models of galaxy formation to offer an interpretation of the results. 


\section{The colour distribution}

Galaxy colours are a simple, useful diagnostic of the galaxy population that can be easily traced to higher redshifts (e.g. Butcher \& Oemler 1984, see also contributions from T. Kodama and F. Nakata in these proceedings). In particular, the fraction of blue galaxies, as defined by a simple, magnitude-dependent colour cut, is known to increase strongly with decreasing galaxy density (e.g. Margoniner \& de Carvalho 2000; De Propris et al. 2004). Using data from the SDSS, Baldry et al. (2004) showed that the galaxy colour distribution is well parametrised by a simple, two-component model where each population is a Gaussian. This allows us to divide the population in an objective way that is insensitive to systematic and statistical uncertainties in the data. The mean and dispersion of each distribution is a strong function of luminosity; in particular, brighter galaxies are redder, an effect that may be partly due to trends in metallicity and dust with stellar mass (Brinchmann et al. 2003).

Recently, we have investigated how these model parameters depend on environment (Balogh et al. 2004a), using the first data release of the SDSS (Abazajian et al. 2003). We select a magnitude-limited sample of 24346 galaxies with $z<0.08$, but we still base our measurement of $\Sigma_{5}$ on the number of galaxies (within a $\pm 1000 \mathrm{~km} \mathrm{~s}^{-1}$ redshift slice) brighter than $M_{r}=-20$ to ensure a uniform definition over the full redshift range.

The dominant trend with environment in the colour distribution is the change in the relative number of galaxies in the blue and red populations. The fraction of red galaxies at fixed luminosity increases from 10-30 per cent to $\sim 70$ per cent toward denser environments. Furthermore, this increase is seen for galaxies at all luminosities, which implies that whatever mechanism is responsible for establishing the correlation must affect bright and faint galaxies in a similar way. We also use the C4 cluster catalogue of Nichol, Miller et al. (in preparation) to test whether the global environment of a surrounding cluster has an additional influence on the colour distribution. Considering all galaxies within the virial radius, the fraction of red galaxies (at fixed luminosity) appears to be independent of velocity dispersion, although the uncertainties are large, due to the relatively small sample of clusters. We conclude, as others have before us (e.g. Dressler 1980; Fairley et al. 2002; De Propris et al. 2004), that the population composition depends primarily on the local environment, and is much less sensitive to the large scale structure.

In contrast, the mean and dispersion of each Gaussian distribution are approximately independent of environment, which is surprising given the large change in their relative abundance. For example, if the increased abundance of red galaxies were due to interactions (e.g. mergers and harassment) that increase in frequency with local galaxy density, we might have expected the blue distribution to become increasingly non-Gaussian with increasing density, and to gradually blend into the red distribution. The fact that this is not observed means that any such transformation must either be sufficiently rapid, or sufficiently rare, to keep the overall colour distribution unchanged. This has the important implication that galaxy clusters do not inhibit star formation in all member galaxies, and we conclude that the properties of star-forming galaxies (dust, metallicity, recent SFR history) are determined primarily by the internal structure of the galaxy, rather than by their environment.

To quantify a limit to the rate of this transformation process, we investigate a simple evolutionary scenario, using the Bruzual \& Charlot (2003) stellar population models, to reproduce the colour distribution of galaxies in dense regions $\left(\Sigma_{5}>1 \mathrm{Mpc}^{-2}\right)$, assuming that the red population results from quenching of star formation in bluer field galaxies of the same luminosity, at a uniform rate over a Hubble time. If the star formation is 
terminated abruptly (the "truncation" scenario) the simple 2-Gaussian model is hardly affected. This is because the colour evolution is rapid, and the number of galaxies caught in transformation at the present day is small ( $\sim 1$ per cent). Therefore, the success of the simple 2-Gaussian model at most luminosities and environments does not rule out the possibility that galaxy transformations occur on short timescales. On the other hand, if star formation is allowed to decay with an exponential timescale $0.5<\tau<4$ Gyr (as expected in the strangulation scenario Larson et al. 1980; Balogh et al. 2000), the number of galaxies with intermediate colours at the present day would increase by a factor $\sim 2$. This would clearly distort the observed distribution in a way that is inconsistent with the observations.

We therefore conclude that short-timescale transformations could play a role at all densities and luminosities, without disrupting the Gaussian model fit. The small fraction of galaxies predicted to be in the transition phase is comparable to the fraction of spectroscopically identified post-starburst galaxies (Goto et al. 2003a; Quintero et al. 2004) which may be the signature of such transformations (e.g. Dressler \& Gunn 1992, see also contributions from A. Dressler, B. Poggianti and K.-V. Tran, in this volume). Another possible candidate is the class of anemic (passively evolving) spirals (van den Bergh 1976; Goto et al. 2003b) which might arise because morphology changes on a longer timescale than the instantaneous SFR. However, we caution that our constraint on the timescale is sensitive to the assumption that the transformations occur uniformly in time. If, instead, transitions were more common in the past, the data would accomodate a slower rate of SFR decay.

\section{Star formation rates}

With uncertainties $\lesssim 1 \AA$ on line equivalent widths, the distribution of $W_{0}(H \alpha)$ also exhibits a bimodality (Gomez et al. 2003). Although the decomposition is not as simply characterised as for the colours, we can approximately divide the sample into star-forming galaxies with $W_{0}(H \alpha)>4 \AA$, and the non-star-forming population with lower equivalent widths. We recently used the 2dFGRS and SDSS to investigate the environmental dependence of this distribution (Balogh et al. 2004b) and found, as expected, that the fraction of galaxies with emission lines declines strongly with increasing density. This correlation persists at all densities, and never asymptotes to a value that is independent of density. Therefore there is no sharp transition, although at densities greater than $\Sigma_{5}=1 \mathrm{Mpc}^{-2}$ the emission-line fraction does begin to decrease more strongly. Interestingly, however, we found that the observed distribution of $W_{0}(H \alpha)$ above $4 \AA$ is independent of local density. Therefore, relative SFRs are not low in clusters, among the star-forming population. This is consistent with the colour analysis, above, and with the fact that both the scatter in the Tully-Fisher relation (e.g. Giuricin et al. 1986; Biviano et al. 1990) and the shape of the UV luminosity function (Cortese et al. 2003) are independent of environment. The result is still surprising, given that these blue galaxies are actively growing and might be thought to be most susceptible to external influences.

Even at the lowest densities, the fraction of emission line (or blue) galaxies is not 100 per cent. We selected a sample of isolated galaxies, based on three criteria. First, we measure the 3-D galaxy density using two smoothing Gaussian kernels with standard deviations of 1.1 and $5.5 \mathrm{Mpc}$, respectively, and select galaxies in the lowest few per cent of both densities. Furthermore, we require that these isolated galaxies do not belong to any group in the comprehensive catalogue of Eke et al. (2004). Even among the brightest of these isolated galaxies, $\sim 30$ per cent show $W_{0}(H \alpha)<4 \AA$, and red colours. Therefore, present-day local environment may not be the only driver of galaxy evolution, since star 
formation has shut off in these isolated galaxies. One possibility is that they are fossil groups (Ponman et al. 1994; Mulchaey \& Zabludoff 1999; Romer et al. 2000), which have completely merged to form a single galaxy.

\section{Model predictions}

The results above could arise in one of at least two ways. One possibility is that the stellar mass of the galaxy determines its properties, and that the stellar mass function varies with environment (as suggested by differences in the $K$-band luminosity functions of cluster and field galaxies, e.g. Balogh et al. 2001; De Propris et al. 2003). The second possibility is that the age distribution at fixed stellar mass depends on environment, implying an environmentally-induced transformation. Kauffmann et al. (2004) have derived stellar masses from the SDSS galaxy sample, and concluded that this is indeed what happens.

Although we do not have stellar masses for the 2dFGRS sample, we can interpret our results by comparison with mock catalogues generated from the updated model predictions of Cole et al. (2000, GALFORM), incorporating the survey selection function and observational biasses. The models we consider are similar to those in Cole et al. (2000) in most respects. The most significant differences are that we use a lower baryon fraction as required by other observations (e.g. Burles et al. 2001) and include the effects of superwind feedback (Benson et al. 2003).

The main success of the model is that it reproduces the observed trend of emissionline fraction on local density extremely well, over two orders of magnitude in density (Figure 1a). Although the agreement in normalisation results partly from the choice of parameters in the dust model, the trend with density is a robust prediction and the good match to the data is remarkable. In Figure $1 \mathrm{~b}$ we show the $W_{0}(H \alpha)$ distribution, for $W_{0}(H \alpha)>4 \AA$, in three environments. Below $\Sigma_{5} \sim 1 \mathrm{Mpc}^{-2}$ (the critical density identified by Lewis et al. 2002), the predicted $W_{0}(H \alpha)$ distribution of star-forming galaxies does not depend on local density, in agreement with the observations in Balogh et al. (2004b). At the highest densities, however, the entire $W_{0}(H \alpha)$ distribution shifts to lower values, in contrast with the observational result.

These predicted trends are not due to a change in the stellar mass function with environment. The superwind feedback limits the number of very massive galaxies by expelling gas from haloes at early times, which results in a mass function that is nearly independent of environment. Therefore the successful prediction of the environmental trend is due to the only transformation mechanism present in these models: strangulation. However, the change in predicted $W_{0}(H \alpha)$ distribution at the highest densities implies that the timescale for this transformation may be shorter than predicted.

\section{Conclusions}

Broadly speaking, galaxy populations are simply characterised by two distinct classes: one that is blue and star-forming, and one that is red and quiescent. The most surprising result of this study is that the colour and $\mathrm{H} \alpha$ distribution of each of these populations is approximately independent of environment in the local Universe. This suggests that SFRs, dust and metallicity distributions are determined primarily by individual galaxy types (e.g. stellar mass) rather than by their surroundings. The fraction of red galaxies increases strongly with increasing density, which is likely the result of transformations that are either rapid, or occur preferentially at high redshift. 

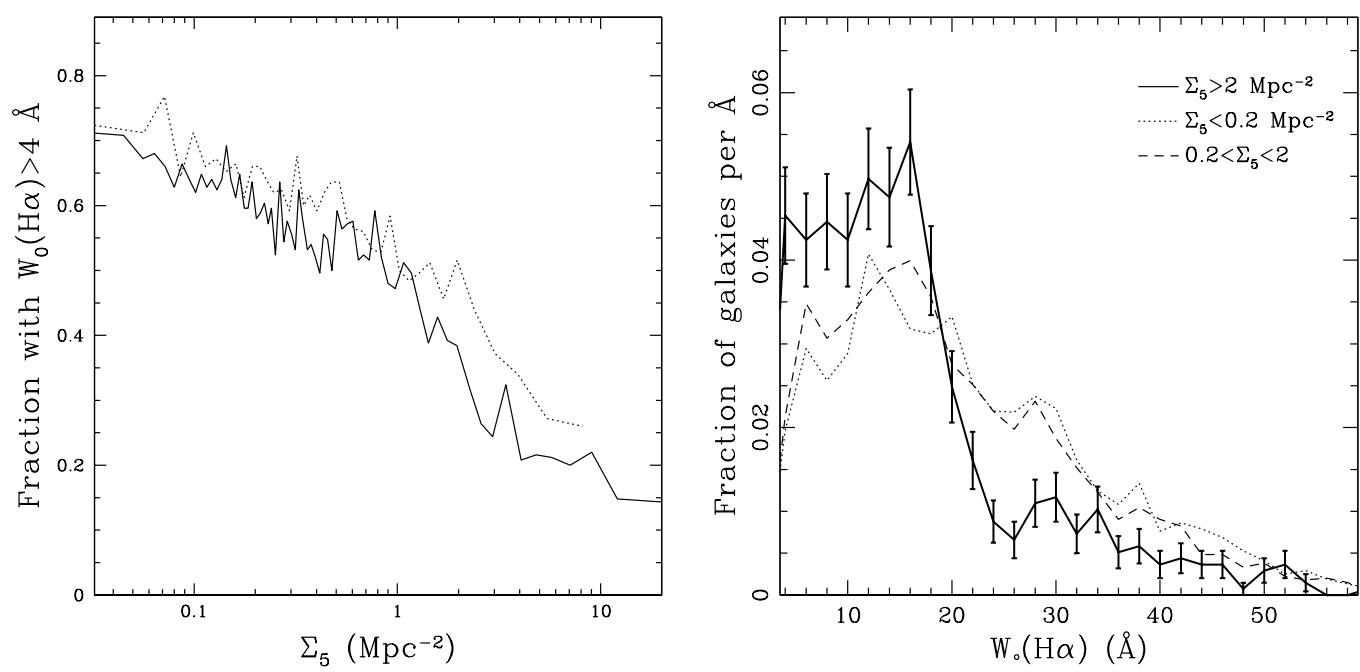

Figure 1. a) Left: The fraction of galaxies with $W_{0}(H \alpha)>4 \AA$, as a function of local, projected density $\Sigma_{5}$. The solid line shows the GALFORM model predictions, and the dashed line represents the 2dFGRS data, from Balogh et al. (2004b). There are 250 galaxies in each bin, so the Poisson-distributed uncertainties are typically $\sim 0.05$. b) Right: The predicted $W_{0}(H \alpha)$ distribution for star-forming galaxies in the GALFORM model, with $W_{0}(H \alpha)>4 \AA$. The solid, dashed and dotted lines represent galaxies in low, intermediate, and high density environments, respectively. We show Poisson-distributed error bars on the high-density subsample, which is the smallest of the three. The change in the shape at highest densities is not observed in the 2dFGRS or SDSS data (Balogh et al. 2004b).

\section{Acknowledgements}

I would like to thank all of my collaborators whose work, both published and unpublished, is represented here. In particular, R. Bower, R. Nichol, I. Baldry, C. Miller, K. Glazebrook, V. Eke, I. Lewis, A. Gray, C. Baugh, S. Cole and C. Frenk and the 2dFGRS team have all contributed to these results.

\section{References}

Abazajian, K. et al. 2003, AJ, 126, 2081

Baldry, I. K., Glazebrook, K., Brinkmann, J., Ivezić, Z., Lupton, R. H., Nichol, R. C., \& Szalay, A. S. 2004, ApJ, 600, 681

Balogh, M. L., Baldry, I. K., Nichol, R. C., Miller, C., Bower, R. G., \& Glazebrook, K. 2004a, ApJL, submitted

Balogh, M. L., Christlein, D., Zabludoff, A. I., \& Zaritsky, D. 2001, ApJ, 557, 117

Balogh, M. L., Morris, S. L., Yee, H. K. C., Carlberg, R. G., \& Ellingson, E. 1997, ApJL, 488, 75

Balogh, M. L., Navarro, J. F., \& Morris, S. L. 2000, ApJ, 540, 113

Balogh, M. L., Schade, D., Morris, S. L., Yee, H. K. C., Carlberg, R. G., \& Ellingson, E. 1998, ApJL, 504, L75

Balogh, M. L. et al. 2004b, MNRAS, 348, 1355

Benson, A. J., Bower, R. G., Frenk, C. S., Lacey, C. G., Baugh, C. M., \& Cole, S. 2003, ApJ, 599,38

Biviano, A., Giuricin, G., Mardirossian, F., \& Mezzetti, M. 1990, ApJS, 74, 325

Brinchmann, J., Charlot, S., et al. 2003, MNRAS, in press, astro-ph/0311060

Bruzual, G. \& Charlot, S. 2003, MNRAS, 344, 1000

Burles, S., Nollett, K. M., \& Turner, M. S. 2001, ApJL, 552, L1 
Butcher, H. \& Oemler, A. 1984, ApJ, 285, 426

Cole, S., Lacey, C. G., Baugh, C. M., \& Frenk, C. S. 2000, MNRAS, 319, 168

Colless, M. et al. 2003, astro-ph/0306581

Cortese, L., Gavazzi, G., Boselli, A., Iglesias-Paramo, J., Donas, J., \& Milliard, B. 2003, A\&A, 410, L25

Cuesta-Bolao, M. J. \& Serna, A. 2003, A\&A, 405, 917

De Propris, R. et al. 2003, MNRAS, 342, 725

—. 2004, MNRAS, in press, astro-ph/0402642

Domínguez, M. J., Zandivarez, A. A., Martínez, H. J., Merchán, M. E., Muriel, H., \& Lambas, D. G. 2002, MNRAS, 335, 825

Dressler, A. 1980, ApJ, 236, 351

Dressler, A. \& Gunn, J. E. 1992, ApJS, 78, 1

Dressler, A., Oemler, A. J., Couch, W. J., Smail, I., Ellis, R. S., Barger, A., Butcher, H., Poggianti, B. M., \& Sharples, R. M. 1997, ApJ, 490, 577

Duc, P.-A., Poggianti, B. M., et al. 2002, A\&A, 382, 60

Eke, V. R. et al. 2004, MNRAS, 348, 866

Fairley, B. W. et al. 2002, MNRAS, 330, 755

Giuricin, G., Mezzetti, M., \& Mardirossian, F. 1986, A\&A, 157, 400

Gomez, P. L., Nichol, R. C., et al. 2003, ApJ, 584, 210

Goto, T., Nichol, R. C., et al. 2003a, PASJ, 55, 771

Goto, T., Okamura, S., et al. 2003b, PASJ, 55, 757

Gray, M. E., Wolf, C., et al. 2004, MNRAS, 347, L73

Gunn, J. E. \& Gott, J. R. 1972, ApJ, 176, 1

Kauffmann, G., White, S. D. M., Heckman, T. M., Menard, B., Brinchmann, J., Charlot, S., Tremonti, C., \& Brinkmann, J. 2004, MNRAS, submitted, astro-ph/0402030

Kennicutt, R. C. 1992, ApJ, 388, 310

Kodama, T. \& Bower, R. G. 2001, MNRAS, 321, 18

Larson, R. B., Tinsley, B. M., \& Caldwell, C. N. 1980, ApJ, 237, 692

Lewis, I., Balogh, M., et al. 2002, MNRAS, 334, 673

Margoniner, V. E. \& de Carvalho, R. R. 2000, AJ, 119, 1562

Margoniner, V. E., de Carvalho, R. R., Gal, R. R., \& Djorgovski, S. G. 2001, ApJL, 548, L143

Marzke, R. O., Geller, M. J., Huchra, J. P., \& Corwin, H. G. 1994, AJ, 108, 437

Moore, B., Lake, G., Quinn, T., \& Stadel, J. 1999, MNRAS, 304, 465

Moss, C. \& Whittle, M. 1993, ApJL, 407, L17

-. 2000, MNRAS, 317, 667

Mulchaey, J. S. \& Zabludoff, A. I. 1999, ApJ, 514, 133

Poggianti, B. M., Bridges, T. J., Komiyama, Y., Yagi, M., Carter, D., Mobasher, B., Okamura, S., \& Kashikawa, N. 2004, ApJ, 601, 197

Poggianti, B. M., Smail, I., Dressler, A., Couch, W. J., Barger, A. J., Butcher, H., Ellis, R. S., \& Oemler, A. J. 1999, ApJ, 518, 576

Ponman, T. J., Allan, D. J., et al. 1994, Nature, 369, 462

Postman, M. \& Geller, M. J. 1984, ApJ, 281, 95

Quintero, A. D., Hogg, D. W., et al. 2004, ApJ, 602, 190

Romer, A. K., Nichol, R. C., et al. 2000, ApJS, 126, 209

Solanes, J., Manrique, A., García-Gómez, C., González-Casado, G., Giovanelli, R., \& Haynes, M. P. 2001a, ApJ, 548, 97

Solanes, J., Manrique, A., García-Gómez, C., et al. 2001b, ApJ, 548, 97

Treu, T., Ellis, R. S., et al. 2003, ApJ, 591, 53

van den Bergh, S. 1976, ApJ, 206, 883

van Gorkom, J. H. 2004, in Clusters of Galaxies: Probes of Cosmological Structure and Galaxy Evolution, from the Carnegie Observatories Centennial Symposia. Published by Cambridge University Press, as part of the Carnegie Observatories Astrophysics Series. Edited by J.S. Mulchaey, A. Dressler, and A. Oemler, 2004, p. 306

Whitmore, B. C., Gilmore, D. M., \& Jones, C. 1993, ApJ, 407, 489

York, D. G., Adelman, J., et al. 2000, AJ, 120, 1579 